\title{
Current classification, treatment options, and new perspectives in the management of adipocytic
}

\section{sarcomas}

This article was published in the following Dove Press journal:

OncoTargets and Therapy

II October 2016

Number of times this article has been viewed

\author{
Alessandro De Vita' \\ Laura Mercatali' \\ Federica Recine' \\ Federica Pieri ${ }^{2}$ \\ Nada Riva' \\ Alberto Bongiovanni' \\ Chiara Liverani' \\ Chiara Spadazzi' \\ Giacomo Miserocchi' \\ Dino Amadori' \\ Toni Ibrahim' \\ 'Osteoncology and Rare Tumors \\ Center, Istituto Scientifico Romagnolo \\ per lo Studio e la Cura dei Tumori \\ (IRST) IRCCS, Meldola, FC, ${ }^{2}$ Pathology \\ Unit, Morgagni-Pierantoni Hospital, \\ Forli, Italy
}

\begin{abstract}
Sarcomas are a heterogeneous group of mesenchymal tumors arising from soft tissue or bone, with an uncertain etiology and difficult classification. Soft tissue sarcomas (STSs) account for around $1 \%$ of all adult cancers. Till date, more than 50 histologic subtypes have been identified. Adipocyte sarcoma or liposarcoma (LPS) is one of the most common STS subtypes, accounting for $15 \%$ of all sarcomas, with an incidence of $24 \%$ of all extremity STSs and $45 \%$ of all retroperitoneal STSs. The new World Health Organization classification system has divided LPS into four different subgroups: atypical lipomatous tumor/well-differentiated LPS, dedifferentiated LPS, myxoid LPS, and pleomorphic LPS. These lesions can develop at any location and exhibit different aggressive potentials reflecting their morphologic diversity and clinical behavior. Patients affected by LPS should be managed in specialized multidisciplinary cancer centers. Whereas surgical resection is the mainstay of treatment for localized disease, the benefits of adjuvant and neoadjuvant chemotherapy are still unclear. Systemic treatment, particularly chemotherapy, is still limited in metastatic disease. Despite the efforts toward a better understanding of the biology of LPS, the outcome of advanced and metastatic patients remains poor. The advent of targeted therapies may lead to an improvement of treatment options and clinical outcomes. A larger patient enrollment into translational and clinical studies will help increase the knowledge of the biological behavior of LPSs, test new drugs, and introduce new methodological studies, that is, on treatment response.
\end{abstract}

Keywords: liposarcomas, adipocytic sarcomas, classification, management

\section{Introduction}

Soft tissue sarcomas (STSs) are an uncommon group of solid neoplasm arising mostly from the embryonic mesoderm of soft tissue, with difficult diagnosis, grading, and management. Even though STSs represent only $1 \%$ of all adult cancers, they exhibit an extraordinary amount of diversity with more than 50 histologic subtypes based on the tumor lineage. ${ }^{1,2}$

Liposarcomas (LPSs) originate from adipocytes and constitute one of the most common STS (15\% of all cases), ${ }^{3}$ with an incidence of $24 \%$ of all extremity STS and $45 \%$ of retroperitoneal STS. ${ }^{4}$ Morphologically, they can be divided into atypical lipomatous tumor or well-differentiated LPS (ALT/WDLPS), dedifferentiated LPS (DDLPS), myxoid LPS (MLPS) (round cell LPS is now considered as a high-grade MLPS and has been removed from the 2013 World Health Organization classification system), and pleomorphic LPS (PLS). ${ }^{5}$

LPS variants exhibit different aggressive potentials reflecting their morphologic diversity. DDLPS, high-grade MLPS, and PLS have a high propensity to metastasize,
Correspondence: Alessandro De Vita Osteoncology and Rare Tumors Center, Istituto Scientifico Romagnolo per lo Studio e la Cura dei Tumori (IRST) IRCCS, Via Piero Maroncelli, 40-470I4 Meldola, FC, Italy Tel +390543739100

Fax +390543739 123

Email alessandro.devita@irst.emr.it 
while ALT/WDLPS does not metastasize without dedifferentiation and MLPS exhibits an indolent clinical behavior and a lower metastatic potential. ${ }^{6}$

LPS can arise at several locations, the retroperitoneum and the extremities being the most common anatomic sites, likely depending on the histologic subtype. Primary MLPS and PLS occur predominantly in the extremities and are very rare in the retroperitoneum, while ALT/WDLPS and DDLPS subtypes arise more often in the retroperitoneum. ${ }^{7-10}$

LPS patients should be managed by specialized multidisciplinary cancer teams. Treatment options involve surgery, chemotherapy (CT), and radiotherapy (RT). The goal of surgery, which represents the standard of care for localized primary tumors, is to achieve complete tumor resection with negative margins, which means completely removing the neoplastic lesion with marginal normal tissue. RT and CT can be administered pre- and/or postoperatively in a multimodal strategy in the management of localized tumors, and have shown controversial results. ${ }^{11}$

The standard treatment of metastatic disease is mostly represented by systemic CT with limited results. Interestingly, LPS sensitivity to CT seems to be correlated to the histologic subtype. In this regard, MLPS has a higher sensitivity to cytotoxic CT than other LPS subtypes. ${ }^{12}$

Recent studies on LPS molecular pathways and genetic mutations have identified new treatment targets with promising results. ${ }^{13,14}$

This paper will provide an overview of LPS, focusing on the current classification system, the different histologic subtypes, and the molecular pathologic features. Finally, treatment options and future directions in clinical care management will be summarized.

\section{Histopathology and clinical behavior}

LPSs are a heterogeneous group of tumors. They can be divided into four distinct subtypes with different clinical behaviors: ALT/WDLPS, DDLPS, MLPS, and PLS LPSs (Table 1).

\section{ALT/WDLPS and DDLPS}

ALT/WDLPS and DDLPS are the most common LPS subtypes representing $40 \%-45 \%$ of all LPSs. Although they usually occur in late adult life, they have been described in all ages. ${ }^{15}$

ALT/WDLPS and DDLPS are characterized by the amplification of 12q13-15 chromosome region which encodes for several potential oncogenes including MDM2, CDK4, HMGIC (also known as HMGA2), SAS, GLI, and DDIT3 (also known as CHOP). ${ }^{16}$ In particular, molecular cytogenetic analysis and immunohistochemistry staining have assessed the prognostic value of CDK4 amplification, which seems to be significantly associated with a worse prognosis in DDLPS (88.2\% in DDLPS vs 58.9\% in ALT/ WDLPS). ${ }^{17}$ From a histologic point of view, the new World Health Organization classification divides ALT/WDLPS into three subclasses according to their morphologic aspect: adipocytic, sclerosing, and inflammatory. Even though three histologic variants are identified, these subclasses have no clinical significance.

Adipocytic ALT/WDLPS is the most frequent subtype which is composed of mature adipocytes with a variation in cell size, focal nuclear atypia, and hyperchromasia, whereas sclerosing LPS exhibits a typical severe nuclear hyperchromasia and rare multivacuolated lipoblasts in an extensive collagenous stroma. Finally, inflammatory ALT/WDLPS is a rare entity characterized by chronic inflammatory infiltration in which the adipocytic nature can be easily overlooked. ${ }^{18}$

DDLPS is characterized by the transition from an adipocyte-rich, well-differentiated region to a nonlipogenic spindle cell-rich region. It could also present either a pleomorphic morphology or the so-called homologous lipoblastic dedifferentiation (PLS like). Most patients develop DDLPS

Table I Summary of some features of LPS

\begin{tabular}{|c|c|c|c|}
\hline Histotypes & $\%$ of incidence in LPS & Diagnosis & Prognosis \\
\hline $\begin{array}{l}\text { ALT: adipocytic, } \\
\text { sclerosing, and } \\
\text { inflammatory }\end{array}$ & $40-45$ together with DDLPS ${ }^{15}$ & FISH analysis of MDM2 ${ }^{16}$ & $\begin{array}{l}\text { Locally aggressive mesenchymal neoplasm. Recurrence is } \\
\text { likely to occur if excision is not complete. }{ }^{20} \text { Progression } \\
\text { from ALT to DDLPS is reported in } 25 \%-40 \% \text { of patients }{ }^{19}\end{array}$ \\
\hline DDLPS & $40-45$ together with $A L T^{15}$ & $\mathrm{FISH}$ analysis of $\mathrm{MDM}^{16}$ & $\begin{array}{l}\text { Strong propensity for distant lung metastases }(10 \%-15 \%) \\
\text { and recurrence }{ }^{20}\end{array}$ \\
\hline MLPS & $30^{22}$ & $\begin{array}{l}\text { FISH analysis of aberrant fusion } \\
\text { gene FUS-CHOP/DDIT } 3^{27}\end{array}$ & $\begin{array}{l}\text { Frequent recurrence. } 10 \%-20 \% \text { of patients develop distant } \\
\text { metastases }{ }^{34}\end{array}$ \\
\hline PLS & $5^{35}$ & Histologic analysis & $\begin{array}{l}\text { Local recurrence in } 30 \%-35 \% \text { of patients; lung is a frequent } \\
\text { site of relapse, but bone and liver metastases have also } \\
\text { been reported }{ }^{37}\end{array}$ \\
\hline
\end{tabular}

Abbreviations: ALT, atypical lipomatous tumor; DDLPS, dedifferentiated liposarcoma; FISH, fluorescence in situ hybridization; LPS, liposarcoma; MDM2, mouse double minute 2; MLPS, myxoid liposarcoma; PLS, pleomorphic liposarcoma. 
de novo, and $25 \%-40 \%$ of patients report progression from ALT/WDLPS to DDLPS. ${ }^{19}$

ALT/WDLPS shows a low metastatic potential, but it is a locally aggressive mesenchymal neoplasm. Tumor growth is slow, but if local excision is not complete, recurrence is likely to occur. DDLPS has a strong propensity for distant lung metastases $(10 \%-15 \%)$ as well as for local recurrences. ${ }^{20}$

Although the etiology of ALT/WDLPS and DDLPS is still unclear, the characteristic presence of 12q13-15 amplification suggests a role in tumor pathogenesis. MDM2 is the most frequent amplified oncogene in ALT/WDLPS and DDLPS, which also acts as a ubiquitin ligase, binding the transactivation domain of the tumor suppressor $\mathrm{p} 53$, thus promoting its degradation. This is confirmed by the fluorescence in situ hybridization analysis of MDM2 gene amplification, representing the standard differential diagnosis of ALT/WDLPS and DDLPS. ${ }^{16}$ In this regard, the presence of both unmutated TP53 and retinoblastoma $(\mathrm{Rb})$ protein in ALT/WDLPS and DDLPS is crucial for the use of molecular therapies as mouse double minute 2 (MDM2) homologue and cyclin dependent kinase-4 (CDK4) antagonists (see discussion below).

The most common site of origin for DDLPS is the retroperitoneum; to a lesser extent, the extremities, the trunk, and the paratesticular area can also be affected. ${ }^{21}$ Usually, dedifferentiation occurs more frequently in retroperitoneal ALT/WDLPS than in extremity LPS, with an incidence of $20 \%$ in the first retroperitoneal local recurrence and $44 \%$ in subsequent local recurrence. ${ }^{19}$

\section{Myxoid liposarcoma}

MLPS is the second most common subtype of LPS accounting for $30 \%$ of all LPS and $\sim 10 \%$ of all adult STSs, ${ }^{22}$ with a tendency to affect lower extremities. Although the median age of patients ranges 50-65 years, MLPS is one of the most common LPS in children and adolescents. ${ }^{23}$

Morphologically, it is characterized by mesenchymal spindle/oval cells organized in a myxoid stroma, with signet ring or multivacuolated lipoblasts and a distinctive plexiform vasculature. MLPS demonstrating the presence of round cell component for more than 5\% has a higher rate of metastasis. Round cell transformation is associated with a worse clinical outcome. ${ }^{24}$ In line with these findings, overall survival (OS) at 5 years is $\sim 90 \%$ in MLPS poor in round cells versus $50 \%$ in MLPS rich in round cells. ${ }^{25}$ In addition to round cell component, high MIB-1 labeling index, p53 missense mutation, reduction of $\mathrm{p} 14$ protein expression and p53 mutation are the adverse prognostic factors. ${ }^{26}$

The most important molecular genetic abnormality of MLPS is the recurrent translocation of the $t(12 ; 16)(q 13 ; p 11)$ region, which results in the aberrant fusion gene, FUS-CHOP/ DDIT3, currently used for the diagnosis of MLPS. ${ }^{27}$ In rare cases, another translocation, (t12;22)(q13;q12), is reported that results in a fusion gene arrangement between EWS and CHOP. It is suggested that both fusion transcripts are involved in the inhibition of normal adipocytic differentiation, leading to the proliferation of immature adipocytes.

FUS-CHOP fusion transcripts are classified into three categories: type I (exons 7-2), type II (exons 5-2), and type III (exons 8-2). ${ }^{28}$ Type II is the most common transcript found in MLPS. The correlation between types of fusion transcripts and clinical behavior is still unclear, but there is strong evidence that these aberrant transcriptional regulators play a central role in primary oncogenesis. ${ }^{29,30}$

Moreover, four types of less-frequent EWS-CHOP fusion transcripts have been identified: type I (exons 7-2), type II (exons 20-2), type III (exons 13-2), and type IV (exons 13-3). The best clinical outcome was described for type I. ${ }^{31,32}$

MLPS usually affects the deep soft tissue of the extremities; the most common site of origin is the thigh, while retroperitoneal and intra-abdominal disease is very rare, usually resulting from a misdiagnosis of ALT/WDLPS and DDLPS. Recurrence is frequently reported, whereas $10 \%-20 \%$ of patients develop distant metastases. MLPS exhibits a special propensity to metastasize to skeletal and pulmonary sites (17\% vs $14 \%),{ }^{33}$ but trunk, chest, and other extremities can also be affected. ${ }^{34}$

Despite receiving appropriate treatment for local disease, nearly $40 \%$ of patients experience disease relapse.

MLPS has a higher sensitivity to cytotoxic CT than other LPS subtypes, ${ }^{12}$ with an OS of 2 years for metastatic disease.

\section{Pleomorphic liposarcoma}

PLS is the least common form of LPS, accounting for only $5 \%$ of cases and occurring mainly in 55-65-year-old patients. ${ }^{35}$ Morphologically, PLS is characterized by a variable number of pleomorphic lipoblasts in a setting of a nonlipogenic highgrade sarcoma, which is necessary for diagnosis. ${ }^{10,36,37}$ A rare epithelioid variant of PLS has also been described. In these cases, positivity for epithelial membrane antigen (26\%) and keratin $(21 \%)$ has been identified. ${ }^{38}$

PLS arises in deep soft tissue, with a particular propensity for the trunk; extremities are less often involved. ${ }^{39}$ PLS is associated with a poor prognosis, high local recurrence, and distant metastasis in $30 \%-35 \%$ of cases. The lung, bone, and liver are the common sites of relapse. ${ }^{37}$ Although PLS rarely occurs in the skin and subcutis, it represents the most common LPS of these sites. Cutaneous and subcutaneous 
PLS exhibit a better outcome than that of deep soft tissue, with $17 \%$ incidence of local recurrence and rare cases of distant metastases. ${ }^{39,40}$

Unlike other LPS, PLS shows a complex karyotype with chromosome arrangements including gains and losses. Consequently, pathogenesis is driven by a complex of events involving genome amplifications and deletions. ${ }^{41}$ Recent studies have hypothesized the role of $\mathrm{p} 14^{\mathrm{ARF}}$ methylation in the origin and growth of PLS. ${ }^{42}$

PLS is the rarest and most aggressive LPS subtype, which is highly resistant to all current treatment options with a very poor clinical outcome. ${ }^{43}$ Its diagnosis is made by histologic analysis.

\section{Treatment options}

Since there are no studies to date on the role of surgery and RT focused specifically on the LPS histotype, this section will report the results of studies performed on STS patients. Recent data are available on histology-driven CT in LPS patients.

\section{Surgery}

Surgical resection of STS, including all LPS histotypes, is the mainstay of therapy for localized disease, with a potentially curative purpose.

In this regard, the principal goal of surgery is resection of the entire tumor with marginal normal tissue. Surgery in STS should be performed by an experienced surgical team.

The most important variables affecting the ability to perform adequate surgery are: the anatomical site, the tumor stage, the histologic subtype, and the tumor grade of sarcoma. ${ }^{44,45}$

\section{Surgery in retroperitoneal sarcoma}

LPS is one of the most common histotypes among retroperitoneal sarcomas. ALT/WDLPS and DDLPS represent the principal histologic subtypes arising in the retroperitoneum, accounting for over $50 \%$ of all retroperitoneal sarcomas. ${ }^{46}$

The prognosis for retroperitoneal sarcomas is poor due to the frequent locoregional recurrence associated with surgery. The complexity of the anatomical site and the tumor size at presentation, with the consequent involvement of adjacent organs and vital structures, are the principal factors correlated to a high incidence of positive surgical margins, which are strong predictors of local recurrence and lower survival. ${ }^{47-49}$

A retrospective study of 500 patients, which was conducted by Lewis et al, showed that complete surgical resection with microscopically negative margins was correlated to a longer survival (103 months) than surgery with macroscopically positive margins (18 months). No difference was found in the unresectable disease. ${ }^{49}$
A high tumor grade is a significant factor of local recurrence and survival, underscoring the relevance of the biology of sarcomas. ${ }^{50}$

Nonetheless, the extension of surgery in STS is still debated, especially in retroperitoneal sarcoma where surgical resection for disease eradication is often challenging.

Recent retrospective data suggest that risk of local recurrence can be lowered through aggressive surgery of retroperitoneal sarcoma, which involves compartmental resection of all adjacent organs. This type of surgical procedure was included in the new surgical guidelines of the European Society for Medical Oncology, ${ }^{11}$ but it is not universally accepted due to the heterogeneity of the retrospective studies, increased surgical morbidity, and conflicting data about the survival benefit of extended surgery. Gronchi et al and Crago et al concluded that extended surgery might be more relevant in low- and intermediate-grade tumors than in highgrade tumors, because in the former, survival is associated primarily with local control..$^{50,51}$

This evidence can be translated into retroperitoneal ALT/ WDLPS, which presents a higher risk of local recurrence and dedifferentiation due to incomplete surgery. On the contrary, DDLPS is associated with a higher risk of distant metastases, thus making the benefit of extended surgery unclear.

However, whether recurrences mainly depend on tumor biology or on adequate surgery is still under debate.

Available data on surgery in retroperitoneal STS are based on retrospective and heterogeneous studies. ${ }^{11,45,52}$

\section{Surgery in extremity sarcoma}

In contrast to the aggressive surgical approach in retroperitoneal STS, the current function-sparing surgery in extremity STS, including LPS, is more conservative, achieving local tumor control with low morbidity and better quality of life. Amputation, once considered the standard surgical treatment in localized STS of the extremities, is now considered only when surgical resection or re-resection with adequate margins cannot be performed without affecting the organ function. ${ }^{53,54}$

The surgical approach in extremity STS changed in the 1980s, thanks to the results of randomized clinical trials comparing amputation with function-sparing surgery and RT. No survival difference between the two treatments was reported, and only $15 \%$ of local recurrence was reported in patients treated with the combination. ${ }^{55}$

However, lower local recurrences were reported after conservative surgery in extremity STS alone. ${ }^{56-59}$ Retrospective analyses have pointed out that surgery alone may apply selectively, emphasizing the relevance of adequate surgical margins as a strong prognostic factor. ${ }^{60,61}$ 
In contrast to these results, a retrospective Italian analysis of 900 STS patients showed that positive surgical margins did not represent an independent factor of prognosis, as it had an unexpected weak adverse prognostic effect. Surgical margins seemed to have greater importance in patients treated with surgery for a local recurrence, as if the local recurrence had selected a subgroup of patients with a more difficult local control, affecting the final outcome. ${ }^{62}$

Although extremity LPS exhibits a high aggressive behavior locally, it has a low metastatic potential unless dedifferentiation occurs.

Further studies are needed to improve selection of patients and personalize surgical approach.

\section{Radiation therapy}

RT can be used in association with surgery in pre- and/or postoperative settings in patients affected by localized STS, including LPS, to improve local tumor control. ${ }^{63,64}$

Despite little is known on the correlation between histology and RT response rates and the lack of clinical trials on LPS, DDLPS and MLPS seem to be more radiosensitive than other LPS subtypes. ${ }^{59,65}$

\section{Radiation therapy in retroperitoneal sarcoma}

Generally, the role of RT is controversial in retroperitoneal tumors.

Postoperative RT can be associated with surgery in retroperitoneal STS to achieve better local control with minimal residual disease, while preoperative RT may be used to improve the resectability of disease. ${ }^{4}$ Till date, no data are available from randomized trials comparing surgery alone with the combined treatment of RT and surgery in retroperitoneal STS. Several retrospective trials have shown that RT in combination with surgery is associated with a lower risk of local relapse in retroperitoneal STSs, while others have recorded no benefit for adjuvant RT. ${ }^{66-69}$

Randomized trials on the benefit of neoadjuvant RT in retroperitoneal STS have failed the accrual of patients. Nevertheless, a preliminary consensus of guidelines was reached for preoperative RT in retroperitoneal STS by a recently published international expert panel, which pointed out that the role of preoperative RT has not been proven yet and that new data are needed. ${ }^{70}$

An ongoing study by The European Organization for Research and Treatment of Cancer (EORTC) is evaluating patients with retroperitoneal STS randomized to en bloc surgery alone versus RT followed by en bloc surgery (STRASS Clinical Trial, Identifier ID: NCT00131898). Abdominal recurrence-free survival is the primary endpoint. Results are still unavailable.

Retrospective trials comparing preoperative versus postoperative RT in patients with retroperitoneal STS have been carried out, whereas randomized trials are still needed. A retrospective analysis conducted by Zagar et al concluded that high dose (60 Gy) of either neoadjuvant or adjuvant RT is safe and efficacious in terms of locoregional control, distant disease-free survival, and OS for treatment of retroperitoneal STS in patients with high risk of residual microscopic or minimal disease at the time of surgery. This study also suggested a possible role of intraoperative RT in the locoregional control of disease with good tolerance. ${ }^{71}$

\section{Radiation therapy in extremity sarcoma}

The role of RT in the treatment of extremity STS is better defined, thanks to two pivotal randomized clinical trials which showed that RT can be an option for the treatment of high-risk, high-grade tumor patients with positive surgical margins, minimizing the risk of local recurrence and preserving the limb function. They both reported a reduced risk in local recurrence with brachytherapy or external beam RT. No improvement in OS or distant failure was reported with RT in extremity STS. ${ }^{64,72}$

Several studies indicated that preoperative RT had similar results to postoperative treatment for local recurrence and survival, although with worse wound complications, which are the most common adverse events in RT. ${ }^{65,71}$ A randomized study conducted by O'Sullivan et al comparing pre- versus postoperative RT in extremity STS showed equivalent local control rates in the management of extremity STS. The trial reported that the incidence of wound complications was related to the timing of RT and that preoperative RT was correlated to a higher risk of wound complications compared to postoperative $\mathrm{RT}^{73}$

The timing and modality of RT remain controversial due to the lack of standardized approaches. Further clinical trials are needed to define patient selection, both in adjuvant and neoadjuvant settings.

For these reasons, RT treatment should be discussed by a multidisciplinary team during treatment decision making.

\section{Cytotoxic agents} Conventional chemotherapeutic agents

CT is the cornerstone of treatment in STS metastatic disease. Neither adjuvant nor neoadjuvant CT is currently a standard therapy for the localized disease.

Anthracyclines were the first systemic chemotherapeutic agents that showed an activity in STS. At present, doxorubicin 
versus doxorubicin-based combination $\mathrm{CT}$ represents the standard first-line therapy in metastatic patients. This regimen can also be used in localized STS as adjuvant or neoadjuvant CT, even though data about survival are conflicting. ${ }^{74-77}$

\section{Adjuvant and neoadjuvant conventional CT in STS}

The most recent meta-analysis on the role of doxorubicin-based CT in the adjuvant setting for resectable STS showed a statistically significant limited improvement in OS (hazard ratio $[\mathrm{HR}]=0.77, P=0.01$ ). The patient population, analyzed from 18 studies using doxorubicin-based CT, was heterogeneous with respect to STS pathologic subtypes and tumor locations. This meta-analysis was not based on individual data. ${ }^{75}$

In a large Phase III randomized clinical trial of the EORTC Soft Tissue and Bone Sarcoma Group, nonmetastatic patients with macroscopically resected grade II-III tumors were randomized to observation or postoperative CT with ifosfamide and doxorubicin with lenograstim. It reported no benefit in either relapse-free survival or OS for resected STS. This trial was not included in the updated meta-analysis. ${ }^{74}$

These results are consistent with a previous EORTC study showing significant benefit in local disease control, but no improvement in OS in patients with resected STS treated with CYVADIC (cyclophosphamide, vincristine, doxorubicin, and dacarbazine) postoperative $\mathrm{CT} .^{78}$

A randomized study conducted by the Italian Sarcoma Group, including patients with high-grade extremity STS treated with postoperative CT with epirubicin and ifosfamide versus observation, showed that disease-free survival and OS were significantly better in the CT group after a 2-year follow-up. Yet, the benefit in OS decreased after a longer follow-up. ${ }^{79}$

Similar disagreements have also been observed in studies on neoadjuvant CT for resectable STS. A randomized Phase II study pointed out that neoadjuvant CT was not associated with better survival in high-risk patients, compared to surgery alone. ${ }^{80}$

An ongoing randomized clinical trial is evaluating standard preoperative CT in extremity STS versus histologydriven CT, involving specific histologic subtypes, including LPS (NCT01710176).

Based on these results, adjuvant and neoadjuvant $\mathrm{CT}$ are not considered standard treatment for localized STS; they should be administered on a case-by-case basis or in clinical trials, as current ESMO guidelines suggest. ${ }^{11}$

\section{Conventional CT in metastatic STS}

In metastatic setting, the recent EORTC 62012 trial, comparing doxorubicin alone versus the combination of doxorubicin and ifosfamide in first-line treatment of advanced or metastatic disease in 455 patients, showed no significant difference in OS between the two groups (28\% vs $31 \%$ OS at 2 years). ${ }^{81}$ In this case series, the proportion of LPS patients was very low (12.5\%). Median progression-free survival (PFS) was significantly higher in the doxorubicin and ifosfamide group versus single-agent doxorubicin (7.4 vs 4.6 months, $P=0.003$ ). A higher objective response rate was observed in the doxorubicin and ifosfamide group than in the doxorubicin group ( $26 \%$ vs $14 \%$ ). As a result, doxorubicin alone represents the standard first-line treatment in advanced LPS patients. Combination treatment can apply if tumor shrinkage is sought to either relieve symptoms or before another intervention. Response of LPS to CT depends on the histologic subtype and the tumor grade, with MLPS exhibiting the highest response rate. ${ }^{12}$

The role of CT in ALT/WDLPS and DDLPS disease was confirmed in a multicenter retrospective study of 208 patients. According to RECIST criteria, the objective response rate was observed in $21 \%$ of patients treated with an anthracycline-containing regimen. PFS at 6 months was seen in 44\% of cases. ${ }^{82}$ Another study on the impact of different single or combination CT regimens in 39 PLS patients reported no significant difference. No differences were observed in terms of objective response rate between anthracycline-containing regimen and non-anthracycline-containing regimen (42\% vs $31 \%, P=0.5$ ) or between single-agent and combination CT $(42 \%$ vs $35 \%, P=0.7)$. PFS at 6 months was $43 \%$ and median OS 14 months, compared to that of other STS subtypes. ${ }^{35}$ Prolonged infusion of ifosfamide in DDLPS patients seemed to yield a higher response rate, as reported in a retrospective study investigating the efficacy of a 14-day infusional ifosfamide regimen in $35 \mathrm{STS}$ patients. DDLPS seemed to have a better response than MLPS and synovial STS, with stable disease observed in $31.8 \%$ of patients. A partial response rate was achieved in $22.7 \%$ of cases. Median PFS was 4.2 months and OS 11.2 months. ${ }^{83}$

The combination of gemcitabine and docetaxel is common in the second-line setting. A randomized Phase II study compared the activity of gemcitabine alone versus the combination of gemcitabine with docetaxel in 122 metastatic STS patients. Twenty of 122 patients were affected by LPS. Stable disease after 24 weeks was reported in $41.6 \%$ of patients treated with gemcitabine alone (5/12 patients) and in $62.5 \%$ with the combination of gemcitabine and 
docetaxel (5/8 patients). A partial response was experienced by two PLS patients. ${ }^{84}$

\section{New agents \\ Marine-derived agents}

\section{Trabectedin}

Trabectedin is a marine alkaloid synthetic DNA-binding compound derived from the Caribbean tunicate Ecteinascidia turbinata. ${ }^{85}$ It covalently binds to the amino groups in the minor groove of the DNA, leading to the formation of DNA-trabectedin complex and subsequent breakage of the double helix, ${ }^{86}$ which results in cell cycle arrest and apoptosis. Trabectedin also seems to exhibit an anti-inflammatory and angiogenesis inhibitory activity, with specific targeting and cytotoxic effect against the macrophages and monocytes associated with the tumor microenvironment. ${ }^{87,88}$ Studies have suggested that decrease in inflammation-mediated factors in the tumor microenvironment might have a role in preventing tumor progression and dissemination. ${ }^{89}$ Trabectedin exhibits specific interactions with the FUS-CHOP fusion protein in MLPS, restoring normal lipoblast differentiation. ${ }^{90}$ Recent studies have focused on the antiangiogenic activity of trabectedin in MLPS. ${ }^{91,92}$ A study of an in vitro model of MLPS clarified the mechanisms of resistance of trabectedin, in which miR-21 and miR-130a seem to be involved, confirming their role as markers in overcoming this mechanism. ${ }^{93}$

A Phase III trial evaluating the efficacy of trabectedin versus doxorubicin-based $\mathrm{CT}$ in the first-line treatment of STS including MLPS showed no significant improvement of trabectedin compared to doxorubicin..$^{94}$

Efficacy and safety were tested in patients affected by advanced or metastatic LPS or leiomyosarcoma (LMS) after failure of treatment with anthracyclines and ifosfamide.

Two different trabectedin schedules were tested: a 24-hour intravenous (IV) infusion once every 3 weeks versus a 3-hour IV infusion every week for 3 weeks in a 4-week cycle. Median time to progression was 3.7 versus 2.3 months, median PFS was 3.3 versus 2.3 months, and median OS was 13.9 versus 11.8 months. The study demonstrated that the 24-hour IV infusion of trabectedin every 3 weeks is well tolerated and effective in this setting of patients. ${ }^{95}$

These data were later confirmed in the expanded access program including 233 LPS cases. ${ }^{96}$ The use of trabectedin in the neoadjuvant setting $\left(1.5 \mathrm{mg} / \mathrm{m}^{2} 24\right.$-hour IV infusion every 3 weeks; $3-6$ cycles) for patients with locally advanced LPS was reported by Gronchi et al. ${ }^{97}$ According to the RECIST criteria, $24 \%$ of patients exhibited an objective response and $13 \%$ showed a pathological complete response; no disease progression was reported and trabectedin was generally well tolerated. Out of 51 MLPS patients of a multicenter retrospective study receiving trabectedin as 24 -hour continuous infusion every 21 days, two patients reported a complete response and 24 patients experienced a partial response, with an overall response of $51 \%$, progression-free rate (PFR) at 3 months of $92 \%$, and PFR at 6 months of $88 \% .^{98}$

Fatigue (8\%) and nausea and vomiting (5\%) represent the most common side effects. Transient neutropenia and rhabdomyolysis are the major, yet rare dose-limiting toxicities. ${ }^{96}$

The efficacy of trabectedin versus dacarbazine was evaluated in a recent Phase III multicenter trial including 518 patients with advanced LPS (27\%) or LMS (73\%) previously treated with an anthracycline and at least one additional systemic therapy. The study showed a $45 \%$ reduction in the risk of progressive disease or death with trabectedin compared to dacarbazine, with a PFS of 4.2 versus 1.5 months and a clinical benefit rate of $34.2 \%$ versus $18.5 \%$, respectively. The safety profiles were consistent with the well-characterized toxicities of both agents. The most common grade 3-4 toxicities in the trabectedin group were neutropenia (40\%) and increased ALT/WDLPS (29\%). ${ }^{99}$

A recently published randomized, open-label, Phase II study of trabectedin after standard CT versus best supportive care (BSC) in Asian patients with advanced translocationrelated STS (including myxoid/round cell LPS, 14/37 versus 10/37 patients) showed a median PFS of 5.6 and 0.07 months in the trabectedin group and the BSC, respectively, with an OS at 1 year of about $70 \%$ in the trabectedin arm (observed for 18 months, median not reached) and a median OS of 8 months in the BSC arm. ${ }^{100}$

An ongoing Phase II, nonrandomized trial, the TR1US study, is evaluating the role of trabectedin in first-line setting patients unfit to receive standard CT (Identifier ID: NCT02066675).

\section{Eribulin mesylate}

Eribulin mesylate, a marine-derived synthetic halichondrin B analog, induces cell cycle arrest and tumor antiproliferative effect by interacting with tubulin protein in cells.

The antiproliferative action of eribulin occurs through a novel tubulin-targeted mechanism that involves binding to a specific site on $\beta$-tubulin and consequent tubulin polymerization.

Eribulin also exerts other complex effects on tumor biology, including vascular remodeling, reversion of the epithelial-mesenchymal transition, and suppression of migration and invasion. ${ }^{101-103}$ 
Eribulin is approved as monotherapy for advanced/ metastatic breast cancer patients. ${ }^{104}$

The activity of this compound is reported in a Phase II study performed by EORTC. A total of 37 patients with LPS were treated with eribulin. PFS at 12 weeks was $46.9 \%$. The most common adverse events included fatigue, cytopenia, neuropathy, and liver function test abnormalities. ${ }^{105}$

Results of a recent randomized, open-label, multicenter Phase III study comparing the efficacy and safety of eribulin versus dacarbazine in patients with STS (adipocytic STS and LMS) in third-line treatment or over showed a better median OS with eribulin than with dacarbazine, that is, 13.5 and 11.5 months, respectively (HR $=0.768,95 \%$ confidence interval $0.618-0.954 ; P=0.017$ ) with a median PFS of 2.6 months in both arms $(\mathrm{HR}=0.877,95 \%$ confidence interval $0.710-1.085$; $P=0.229$ ). In the preplanned OS subgroup analysis, median OS was higher in adipocytic STS (15.6 vs 8.4 months) than in LMS (12.7 vs 13 months). Neutropenia (44\% vs 24\%), fever ( $28 \%$ vs $14 \%$ ), peripheral sensory neuropathy ( $20 \%$ vs $4 \%$ ), and alopecia ( $35 \%$ vs $3 \%$ ) were reported in both the eribulin and dacarbazine arms. Thrombocytopenia was more frequent with dacarbazine treatment ( $28 \%$ vs $6 \%)$. ${ }^{106,107}$

These results have led to the recent Food and Drug Administration approval of eribulin for patients with metastatic LPS who have received prior $\mathrm{CT}$ with anthracyclines.

\section{Molecular therapies}

Tyrosine kinase receptor inhibitors: pazopanib, sorafenib, sunitinib, and dasatinib

Tyrosine kinase receptors are a family of high-affinity cell surface receptors for growth factors, cytokines, and hormones, which play a pivotal role in carcinogenesis, angiogenesis, and metastasis formation. Extracellular ligand binding typically causes or stabilizes receptor dimerization, which results in the activation of the intracellular signaling pathway. Overexpression of these receptors in many tumor subtypes makes them potential candidates for cancer treatment, in particular, STSs. ${ }^{108}$ Pazopanib is a novel small-molecule, multitargeted tyrosine kinase inhibitor (TKI) that is active against vascular endothelial growth factor (VEGF) 1, 2, and 3, platelet-derived growth factor (PDGF), and KIT. ${ }^{109}$ The activity of TKI on advanced STS was assessed in a 142-patient Phase II trial. As insufficient activity was seen in the LPS patients, enrollment was stopped after the first stage. Final results showed that PFR at 12 weeks was $26 \%$ in five out of 19 LPS patients. ${ }^{110}$ Another Phase II study evaluating the activity of pazopanib in advanced LPS patients is still ongoing (NCT01506596).
Sorafenib is another multitargeted TKI which exhibits activity against VEGF 1, 2, and 3, B-Raf, C-Raf, PDGF, KIT, and Fms-related tyrosine kinase $3 .{ }^{111}$ A Phase II clinical trial testing sorafenib activity in patients with different types of STS including LPS was carried out by von Mehren et al. ${ }^{112}$ Ten LPS patients (eight with DDLPS and two with MLPS) received sorafenib at a dose of $400 \mathrm{mg}$ twice daily. The authors reported no response meeting the RECIST criteria, although two DDLPS patients showed disease stabilization. Another sorafenib Phase II trial is reported by Maki et al, with different STS histologies: 145 patients with different STS were included, but only three patients were affected by LPS. ${ }^{113}$

Sunitinib is another TKI inhibitor active against PDGF, VEGF, KIT, RET, Fms-related tyrosine kinase 3, and CSF3. ${ }^{114}$ A Phase II trial of sunitinib in 48 patients with relapsed or refractory STS was reported by Mahmood et al. ${ }^{115}$ Sunitinib malate was administered at a dose of $50 \mathrm{mg}$ daily for 4 weeks every 6 weeks. Fourteen out of 17 LPS patients (82\%) experienced stable disease, and PFR at 3 months was $63 \%$.

Dasatinib is a small-molecule inhibitor of PDGF, SRC, KIT, BCR-ALB, and ephrin receptor kinases. A Phase II study of dasatinib failed to demonstrate a clinically significant activity in patients previously treated for high grade metastatic STS, including LPS. ${ }^{116}$

The few clinical trials available, limited number of patients enrolled, and the often unmentioned histologic LPS subtypes confirm the need for further investigation to better understand the mechanism and efficacy of these molecules in the treatment of LPS patients.

\section{MDM2 antagonists}

MDM2, also known as E3 ubiquitin-protein ligase, acts as an antagonist of the tumor suppressor p53. Inactivation of p53 pathway results in the inhibition of cell cycle arrest and apoptosis. Amplification of MDM2 is seen in many tumors, especially in ALT/WDLPS and DDLPS. For this reason, the presence of MDM2 amplification is a useful tool for diagnosis in these LPS histologic subgroups. ${ }^{36}$ Moreover, inhibition of MDM2 interaction with $\mathrm{p} 53$ tumor suppressor protein represents a new strategic approach to cancer treatment. A class of imidazoline agents discovered by Vassilev et al, termed nutlins, binds MDM2 in the 553 pocket and strongly inactivates p53-MDM2 interaction, restoring p53 functions. ${ }^{117}$ Preclinical studies on the molecular response of nutlin- $3 \mathrm{a}$, the most active MDM2 inhibitor, reported a promising activity in ALT/WDLPS and DDLPS. Nutlin-3a efficiently stabilized 
p53 and induced downstream p53-dependent transcription and apoptosis in LPS. ${ }^{118,119}$

The first clinical study to have employed the oral MDM2 antagonist RG7112 was recently performed by Ray-Coquard et al. ${ }^{120}$ This Phase I clinical trial assessed the toxicity of RG7112 in patients with different solid tumors, including LPS. This pilot study enrolled 20 patients with localized and resectable LPS, with the doses tested being $20-1,800 \mathrm{mg} / \mathrm{m}^{2}$. RG7112 was given in the neoadjuvant setting prior to surgery in three 28-day cycles. Although RG7112 was well tolerated, out of 20 patients, six experienced grade 4 neutropenia, three experienced thrombocytopenia, and almost all patients had nausea, vomiting, and fatigue. The results, based on RECIST criteria, reported one partial remission and 14 stable diseases. Analysis of resected tumors confirmed the restoration of p53 and downstream p21 expression in KI67-positive cells. TUNEL analysis showed an increase in apoptotic tumor cells in the MDM2 antagonist treatment.

Further studies are needed to identify the optimal standard dose and reduce the adverse events.

\section{CDK4 antagonists}

CDK4 is a protein kinase that plays a crucial role in primary oncogenesis by inhibition of $\mathrm{p} 53$ activity and by promoting cell cycle progression by $\mathrm{Rb}$ protein phosphorylation. CDK4 inactivation may represent a potential approach to cancer therapy.

CDK4 is frequently amplified in many ALT/WDLPSs and DDLPSs; its absence is associated with favorable prognosis in LPS. ${ }^{121,122}$

Preclinical studies in STS have shown that CDK4 inhibitors may act as potentiators of cytotoxic CT agent, doxorubicin. ${ }^{123}$ A Phase I trial of the selective oral CDK inhibitor, seliciclib, enrolling 21 patients with advanced solid tumors reported no objective tumor response and stable disease in eight patients. Grade 3 toxicities including fatigue, skin rash, and hyponatremia were reported. Other toxicities included grade 4 hypokalemia, raised creatinine, grade 2 emesis, and reversible abnormal liver function grade 3.124 An open-label Phase II trial of the oral CDK4 inhibitor PD0332991 was carried out by Dickson et al. ${ }^{125}$ The study enrolled 48 patients with advanced ALT/WDLPS and DDLPS (44 of 48 had CDK 4 amplification; 41 of 44 were $\mathrm{Rb}$ positive). PD0332991 $200 \mathrm{mg}$ was given orally once daily for 14 consecutive days in a 21-day cycle. PFS at 12 weeks was $66 \%$, one partial response was reported, and patients were able to achieve prolonged stable disease. Although the treatment schedule was well tolerated, PD0332991 was associated with hematological toxicity including anemia $(17 \%)$, thrombocytopenia $(30 \%)$, neutropenia $(50 \%)$, and febrile neutropenia (3\%). PFS rates suggested a promising role of this CDK4 inhibitor in advanced ALT/WDLPS and DDLPS. Other CDK4 inhibitors, including LY2835219 and LEE001, are currently under evaluation in clinical trials. These agents showed different profiles of toxicity; gastrointestinal adverse effects were associated with LY2835219, and neutropenia and QT prolongation were mostly recorded with LEE001. LPS seems to be responsive to these agents, but definitive results are still unavailable. ${ }^{126,127}$

\section{Peroxisome proliferator-activated receptor gamma agonists}

Peroxisome proliferator-activated receptor gamma (PPAR- $\gamma$ ) plays a role in the terminal adipocyte differentiation pathway. In vitro studies have revealed antitumor activity of PPAR- $\gamma$ agonists in LPS cell lines. ${ }^{128,129}$ Activation of PPAR- $\gamma$ may represent a promising tool, not only for cancer treatment but also for reverting more aggressive LPS phenotypes to welldifferentiated ones with better clinical outcomes.

Demetri et al conducted a clinical trial on advanced LPS (two MLPS and one PLS) treated with troglitazone, a PPAR- $\gamma$ ligand. ${ }^{129}$ Histologic and immunohistochemical analysis showed strong evidence of extensive lipid accumulation by tumor cells. Yet, no clinical benefit was observed in 12 DDLPS and MLPS patients receiving troglitazone in a Phase II trial. ${ }^{130}$ Promising results were reported in a more recent Phase I clinical trial in which the safety and the maximum tolerated dose of efatutazone (CS-7017), a novel PPAR- $\gamma$ agonist, were tested. Five out of 31 patients had LPS; efatutazone was administered orally twice daily for 6 weeks. Peripheral edema was experienced by the majority of patients (53.3\%), and three episodes of dose-limiting toxicities were observed. Results reported a sustained partial response for 690 days of therapy in one case of MLPS. ${ }^{131}$

These data suggest that PPAR- $\gamma$ agonists may have a role in the management of LPS patients, although further studies are needed to confirm it.

\section{Nelfinavir}

Nelfinavir is an HIV protease inhibitor showing promising anticancer activity by binding to sterol regulatory elementbinding protein-1 (SREBP-1), which plays a role in lipogenic expression. Alterations in SREBP-1 expression result in a clinical syndrome of lipodystrophy, which is characterized by abnormal adopigenesis, peripheral fat atrophy, and bizarre lipid distribution. ${ }^{132}$ 
In a Phase I study assessing the tolerability of nelfinavir on LPS, 20 patients with unresectable disease were treated twice daily with a dose escalation starting from 1.250 to $4.250 \mathrm{mg}$. Seventeen patients had DDLPS and ALT/WDLPS, two had MLPS, and one patient had PLS. A partial response was observed in one DDLPS patient for 14 months; four patients experienced stable disease. No dose-limiting toxicity was reported after treatment with nelfinavir; only one patient exhibited grade 3 pancreatitis. ${ }^{133}$ Further studies are needed.

\section{Other targeted therapies}

Some other targeted therapies have been studied in the treatment of STS, but have shown no benefit.

Bevacizumab, a human monoclonal antibody binding human VEGF, has been investigated in STS. A Phase II trial evaluated the safety and efficacy of gemcitabine and docetaxel with the addition of bevacizumab versus gemcitabine and docetaxel showed an improvement in PFS and response rate at 3 months (not confirmed at 6 months) in 35 patients with STS including PLS. ${ }^{134}$

Antiepidermal growth factor receptor cetuximab showed no activity as a single agent in STS. ${ }^{135}$

\section{Future directions}

Recent findings have focused on genes like ZIC1, TOP2A, and AURKA, which seem to have high expression levels across LPS,${ }^{4}$ becoming promising candidate targets for new therapy and diagnosis.

The recently discovered oncoprotein, gankyrin, is overexpressed in ALT/WDLPS and DDLPS. This antiapoptotic protein represents a promising predictive oncogenic factor in these two types of LPS. ${ }^{136}$

Recent efforts focus on the promising results of immunotherapy in several tumor types including STS, despite its limited role in STS.

Clinical trials on the role of pembrolizumab, an IgG4- $\kappa$ humanized monoclonal antibody that blocks PD-1, are ongoing. A Phase I trial demonstrated that pembrolizumab is associated with good tolerability and durable antitumor activity in patients with multiple solid tumors including STS. ${ }^{137}$ A Phase II trial on the role of pembrolizumab in STS including LPS is ongoing (NCT02301039).

An open-label Phase II randomized study is currently evaluating CMB305 and atezolizumab in patients with STS including LPS (NCT02609984).

\section{Conclusion}

STSs, consisting of a heterogeneous group of more than 50 histotypes, are rare, but often fatal diseases due to their tendency to metastasize. LPS is one of the most common STS subtypes. Till date, surgery is the mainstay of treatment for localized disease, while the role of adjuvant and neoadjuvant CT for localized STS is still controversial. In metastatic disease, systemic CT is the standard of treatment and has shown limited results.

A multidisciplinary approach involving oncologists, surgeons, pathologists, radiotherapists, and radiologists is needed for the management of the disease.

More data has recently been reported on novel treatments. Preliminary results of some of these systemic drugs with different molecular targets are encouraging, with expected improvements in clinical outcome.

A larger patient enrollment into translational and clinical studies is crucial to better understand the biology of these tumors and develop new therapeutic strategies, which can have an impact on the treatment of LPS patients.

Further evaluations are needed to translate these new treatment options into daily clinical practice.

\section{Acknowledgment}

The authors would like to thank Cristiano Verna and Veronica Zanoni for editorial assistance.

\section{Author contributions}

All authors contributed toward data analysis, drafting and revising the paper and agree to be accountable for all aspects of the work.

\section{Disclosure}

The authors report no conflicts of interest in this work.

\section{References}

1. Siegel RL, Miller KD, Jemal A. Cancer statistics. CA Cancer J Clin. 2016;66(1):7-30.

2. Linch M, Miah AB, Thway K, Judson IR, Benson C. Systemic treatment of soft-tissue sarcoma gold standard and novel therapies. Nat Rev Clin Oncol. 2014;11(4):187-202.

3. Ducimetière F, Lurkin A, Ranchère-Vince $D$, et al. Incidence of sarcoma histotypes and molecular subtypes in a prospective epidemiological study with central pathology review and molecular testing. PLoS One. 2011;6(8):e20294.

4. Crago AM, Singer S. Clinical and molecular approaches to well differentiated and dedifferentiated liposarcoma. Curr Opin Oncol. 2011; 23(4):373-378.

5. Fletcher CDM. The evolving classification of soft tissue tumours - an update based on the new 2013 WHO classification. Histopathology. 2014;64(1):2-11.

6. Rieker RJ, Weitz J, Lehner B, et al. Genomic profiling reveals subsets of dedifferentiated liposarcoma to follow separate molecular pathways. Virchows Arch. 2010;456(3):277-285.

7. Lewis JJ, Leung D, Heslin M, Woodruff JM, Brennan MF. Association of local recurrence with subsequent survival in extremity soft tissue sarcoma. J Clin Oncol. 1997;15(2):646-652. 
8. de Vreeze RS, de Jong D, Tielen IH, et al. Primary retroperitoneal myxoid/round cell liposarcoma is a nonexisting disease: an immunohistochemical and molecular biological analysis. Mod Pathol. 2009;22(2): 223-231.

9. Sioletic S, Dal Cin P, Fletcher CD, Hornick JL. Well-differentiated and dedifferentiated liposarcomas with prominent myxoid stroma: analysis of 56 cases. Histopathology. 2013;62(2):287-293.

10. Hornick JL, Bosenberg MW, Mentzel T, McMenamin ME, Oliveira AM, Fletcher CD. Pleomorphic liposarcoma: clinicopathologic analysis of 57 cases. Am J Surg Pathol. 2004;28(10):1257-1267.

11. The ESMO/European Sarcoma Network Working Group. Soft tissue and visceral sarcomas: ESMO Clinical Practice Guidelines for diagnosis, treatment and follow-up. Ann Oncol. 2014:25(Suppl 3):iii102-iiil12.

12. Jones RL, Fisher C, Al-Muderis O, Judson IR. Differential sensitivity of liposarcoma subtypes to chemotherapy. Eur J Cancer. 2005;41(18): 2853-2860.

13. Guan Z, Yu X, Wang H, et al. Advances in the targeted therapy of liposarcoma. Onco Targets Ther. 2015;8:125-136.

14. Barretina J, Taylor BS, Banerji S, et al. Subtype-specific genomic alterations define new target for soft-tissue sarcoma therapy. Nat Genet. 2010;42(8):715-721.

15. Schmidt H, Bartel F, Kappler M, et al. Gains of $13 q$ are correlated with a poor prognosis in liposarcoma. Mod Pathol. 2005;18(5):638-644.

16. Hostein I, Pelmus M, Aurias A, Pedeutour F, Mathoulin-Pélissier S, Coindre JM. Evaluation of MDM2 and CDK4 amplification by real-time PCR on paraffin wax-embedded material: a potential tool for the diagnosis of atypical lipomatous tumours/well-differentiated liposarcomas. J Pathol. 2004;202(1):95-102.

17. Saâda-Bouzid E, Burel-Vandenbos F, Ranchère-Vince D. Prognostic value of HMGA2, CDK4, and JUN amplification in well-differentiated and dedifferentiatedliposarcomas. Mod Pathol. 2015;28(11):1404-1414.

18. Dei Tos AP. Liposarcoma: new entities and evolving concepts. Ann Diagn Pathol. 2000;4(4):252-266

19. Singer S, Antonescu CR, Riedel E, Brennan MF. Histologic subtype and margin of resection predict pattern of recurrence and survival for retroperitoneal liposarcoma. Ann Surg. 2003;238(3):358-370.

20. Ghadimi MP, Al-Zaid T, Madewell J, Peng T, Colombo C. Diagnosis, management, and outcome of patients with dedifferentiated liposarcoma systemic metastasis. Ann Surg Oncol. 2011;18(13):3762-3770.

21. Tseng WW, Somaiah N, Lazar AJ, Lev DC, Pollock RE. Novel systemic therapies in advanced liposarcoma: a review of recent clinical trial results. Cancers (Basel). 2013;5(2):529-549.

22. Conyers R, Young S, Thomas DM. Liposarcoma: molecular genetics and therapeutics. Sarcoma. 2011;2011:483154.

23. Huh WW, Yuen C, Munsell M, et al. Liposarcoma in children and young adult: a multi-istitutional experience. Pediatr Blood Cancer. 2011;57(7): 1142-1146.

24. Fiore M, Grosso F, Lo Vullo S, et al. Myxoid/round cell and pleomorphic liposarcomas: prognostic factors and survival in a series of patients treated at a single institution. Cancer. 2007;109(12):2522-2531.

25. Antonescu CR, Tschernyavsky SJ, Decuseara R, et al. Prognostic impact of P53 status, TLS-CHOP fusion transcript structure, and histological grade in myxoid liposarcoma: a molecular and clinicopathologic study of 82 cases. Clin Cancer Res. 2001;7(12):3977-3987.

26. Oda Y, Yamamoto H, Takahira T, et al. Frequent alteration of $\mathrm{p} 16(\mathrm{INK} 4 \mathrm{a}) / \mathrm{p} 14(\mathrm{ARF})$ and $\mathrm{p} 53$ pathways in the round cell component of myxoid/round cell liposarcoma: p53 gene alterations and reduced p14(ARF) expression both correlate with poor prognosis. J Pathol. 2005; 207(4):410-421.

27. Narendra S, Valente A, Tull J, Zhang S. DDIT3 gene break-apart as a molecular marker for diagnosis of myxoid liposarcoma-assay validation and clinical experience. Diagn Mol Pathol. 2011;20(4):218-224.

28. Nishio J, Iwasaki H, Nabeshima K, Naito M. Cytogenetics and molecular genetics of myxoid soft-tissue sarcomas. Genet Res Int. 2011; 2011:497148.

29. Pérez-Mancera PA, Sánchez-García I. Understanding mesenchymal cancer: the liposarcoma-associated FUS-DDIT3 fusion gene as a model. Semin Cancer Biol. 2005;15(3):206-214.
30. Kuroda M, Ishida T, Takanashi M, Satoh M, Machinami R, Watanabe T. Oncogenic transformation and inhibition of adipocytic conversion of preadipocytes by TLS/FUS-CHOP type II chimeric protein. Am J Pathol. 1997;151(3):735-744.

31. Suzuki K, Matsui Y, Endo K, et al. Myxoid liposarcoma with EWS-CHOP type 1 fusion gene. Anticancer Res. 2010;30(11):4679-4683.

32. Matsui Y, Ueda T, Kubo T, et al. A novel type of EWS-CHOP fusion gene in myxoid liposarcoma. Biochem Biophys Res Commun. 2006; 348(2):437-440.

33. Schwab JH, Boland PJ, Antonescu C, Bilsky MH, Healey JH. Spinal metastases from myxoid liposarcoma warrant screening with magnetic resonance imaging. Cancer. 2007;110(8):1815-1822.

34. Asano N, Susa M, Hosaka S, et al. Metastatic patterns of myxoid/round cell liposarcoma: a review of a 25-year experience. Sarcoma. 2012; 2012:345161.

35. Italiano A, Garbay D, Cioffi A, Maki RG, Bui B. Advanced pleomorphic liposarcomas: clinical outcome and impact of chemotherapy. Ann Oncol. 2012;23(8):2205-2216.

36. Dodd LG. Update on liposarcoma: a review for cytopathologists. Diagn Cytopathol. 2012;40(12):1122-1131.

37. Downes KA, Goldblum JR, Montgomery EA, Fisher C. Pleomorphic liposarcoma: a clinicopathologic analysis of 19 cases. Mod Pathol. 2001;14(3):179-184.

38. De Cecco L, Gariboldi M, Reid JF, et al. Gene expression profile identifies a rare epithelioid variant case of pleomorphic liposarcoma carrying FUS-CHOP transcript. Histopathology. 2005;46(3):334-341.

39. Gardner JM, Dandekar M, Thomas D, et al. Cutaneous and subcutaneous pleomorphic liposarcoma: a clinicopathologic study of 29 cases with evaluation of MDM2 gene amplification in 26. Am J Surg Pathol. 2012;36(7):1047-1051.

40. Dei Tos AP, Mentzel T, Fletcher CD. Primary liposarcoma of the skin: a rare neoplasm with unusual high grade features. Am J Dermatopathol. 1998;20(4):332-338.

41. Idbaih A, Coindre JM, Derré J, et al. Myxoid malignant fibrous histiocytoma and pleomorphic liposarcoma share very similar genomic imbalances. Lab Invest. 2005;85(2):176-181.

42. Davidović R, Sopta J, Mandušić V, et al. p14(ARF) methylation is a common event in the pathogenesis and progression of myxoid and pleomorphic liposarcoma. Med Oncol. 2013;30(3):682.

43. Ghadimi MP, Liu P, Peng T, et al. Pleomorphic liposarcoma: clinical observations and molecular variables. Cancer. 2011;117(23):5359-5369.

44. Matthyssens LE, Creytens D, Ceelen P. Retroperitoneal liposarcoma: current insights in diagnosis and treatment. Front Surg. 2015;2:4.

45. Gronchi A, Lo Vullo S, Fiore M, et al. Aggressive surgical policies in a retrospectively reviewed single-institution case series of retroperitoneal soft tissue sarcoma patients. J Clin Oncol. 2009;27(1):24-30.

46. Dalal KM, Kattan MW, Antonescu CR, Brennan MF, Singer S. Subtype specific prognostic nomogram for patients with primary liposarcoma of the retroperitoneum, extremity, or trunk. Ann Surg. 2006;244(3): 381-391.

47. Lewis JJ, Brennan MF. Soft tissue sarcomas. Cuff Probl Surg. 1996; 33(10):817-872.

48. Brennan MF, Antonescu CR, Moraco N, Singer S. Lessons learned from the study of 10.000 patients with soft tissue sarcoma. Ann Surg. 2014; 260(3):416-421.

49. Lewis JJ, Leung D, Woodruff JM, Brennan MF. Retroperitoneal softtissue sarcoma: Analysis of 500 patients treated and followed at a single institution. Ann Surg. 1998;228(3):355-365.

50. Gronchi A, Miceli R, Colombo C, et al. Frontline extended surgery is associated with improved survival in retroperitoneal low-to intermediategrade soft tissue sarcomas. Ann Oncol. 2012;23(4):1067-1076.

51. Crago AM. Extended surgical resection and histology in retroperitoneal sarcoma. Ann Sur Oncol. 2015;22(5):1401-1403.

52. Gronchi A, Casali PG, Fiore M, et al. Retroperitoneal soft tissue sarcomas. patterns of recurrence in 167 patients treated at a single institution. Cancer. 2004;100(11):2448-2455.

53. Clark MA, Thomas JM. Amputation for soft-tissue sarcoma. Lancet Oncol. 2003;4(6):335-342. 
54. Lin PP, Guzel VB, Pisters PW, et al. Surgical management of soft tissue sarcomas of the hand and foot. Cancer. 2002;95(4):852-861.

55. Rosenberg SA, Tepper J, Glatstein E, et al. The treatment of soft-tissue sarcomas of the extremities: prospective randomized evaluations of (1) limb-sparing surgery plus radiation therapy compared with amputation and (2) the role of adjuvant chemotherapy. Ann Surg. 1982; 196(3):305-315.

56. Leibel SA, Tranbaugh RF, Wara WM, Beckstead JH, Bovill EG, Phillips TL. Soft tissue sarcomas of the extremities. Cancer. 1982;50(6): 1076-1083.

57. Karakousis CP, Emrich LJ, Rao U, Krishnamsetty RM. Feasibility of limb salvage and survival in soft tissue sarcomas. Cancer. 1986;57(3): 484-491.

58. Suit HD, Mankin HJ, Wood WC, et al. Treatment of the patient with stage M0 soft tissue sarcoma. J Clin Oncol. 1988;6(5):854-862.

59. Singer S, Corson JM, Gonin R, Labow B, Eberlein TJ. Prognostic factors predictive of survival and local recurrence for extremity soft tissue sarcoma. Ann Surg. 1994;219(2):165-173.

60. Baldini EH, Goldberg J, Jenner C, et al. Long-term outcomes after function-sparing surgery without radiotherapy for soft tissue sarcoma of the extremities and trunk. J Clin Oncol. 1999;17(10):3252-3259.

61. Rydholm A, Gustafson P, Rooser B, et al. Limb-sparing surgery without radiotherapy based on anatomic location of soft tissue sarcoma. J Clin Oncol. 1991;9(10):1757-1765.

62. Gronchi A, Casali PG, Mariani L, et al. Status of surgical margins and prognosis in adult softtissue sarcomas of the extremities: a series of patients treated at a single Institution. $J$ Clin Oncol. 2005;23(1): 96-104.

63. Petersen IA, Haddock MG, Donohue JH, et al. Use of intraoperative electron beam radiotherapy in the management of retroperitoneal soft tissue sarcomas. Int J Radiat Oncol Biol Phys. 2002;52(2):469-475.

64. Pisters PW, Harrison LB, Leung DH, Woodruff JM, Casper ES, Brennan MF. Long-term results of a prospective randomized trial of adjuvant brachytherapy in soft tissue sarcoma. J Clin Oncol. 1996;14(3): 859-868.

65. Chung PW, Deheshi BM, Ferguson PC, et al. Radiosensitivity translates into excellent local control in extremity myxoid liposarcoma: A comparison with other soft tissue sarcomas. Cancer. 2009;115(14): 3254-3261.

66. Sampath S, Hitchcock YJ, Shrieve DC, Randall RL, Schultheiss TE, Wong JY. Radiotherapy and extent of surgical resection in retroperitoneal soft-tissue sarcoma: multi-institutional analysis of 261 patients. J Surg Oncol. 2010;101(5):345-350.

67. Stoeckle E, Coindre JM, Bonvalot S, et al. Prognostic factors in retroperitoneal sarcoma: a multivariate analysis of a series of 165 patients of the French Cancer Center Federation Sarcoma Group. Cancer. 2001; 92(2):359-368.

68. Van Doorn RC, Gallee MP, Hart AA, et al. Resectable retroperitoneal soft tissue sarcomas. The effect of extent of resection and post-operative radiation therapy on local tumor control. Cancer. 1994;73(3):637-642.

69. Catton CN, O' Sullivan B, Kotwall C, Cummings B, Hao Y, Fornasier V. Outcome and prognosis in retroperitoneal soft tissue sarcoma. Int $J$ Radiat Oncol Biol Phys. 1994;29(5):1005-1010.

70. Baldini EH, Wang D, Haas RL, et al. Treatment guidelines for preoperative radiation therapy for retroperitoneal sarcoma: preliminary consensus of an international expert panel. Int J Radiat Oncol Biol Phys. 2015; 92(3):602-612.

71. Zagar MT, Shenk RR, Kim JA, et al. Radiation therapy in addition to gross total resection of retroperitoneal sarcoma results in prolonged survival: results from a single institutional study. J Oncol. 2008; 2008:824036.

72. Yang JC, Chang AE, Sindelar WF, et al. Randomized prospective study of the benefit of adjuvant radiation therapy in the treatment of soft tissue sarcomas of the extremity. J Clin Oncol. 1998;16(1):197-203.

73. O'Sullivan B, Davis AM, Turcotte R, et al. Preoperative versus postoperative radiotherapy in soft-tissue sarcoma of the limbs: a randomized trial. Lancet. 2002;359(9325):2235-2224.
74. Woll PJ, Reichardt P, Le Cesne A, et al. EORTC Soft Tissue and Bone Sarcoma Group and the NCIC Clinical Trials Group Sarcoma Disease Site Committee. Adjuvant chemotherapy with doxorubicin, ifosfamide, and lenograstim for resected soft-tissue sarcoma (EORTC 62931): a multicentre randomised controlled trial. Lancet Oncol. 2012;13(10): $1045-1105$.

75. Pervaiz N, Colterjohn N, Farrokhyar F, Tozer R, Figueredo A, Ghert M. A systematic meta-analysis of randomized controlled trials of adjuvant chemotherapy for localized resectable soft-tissue sarcoma. Cancer. 2008;113(3):573-577.

76. Miura JT, Charlson J, Gamblin TC, et al. Impact of chemotherapy on survival in surgically resected retroperitoneal sarcoma. Eur J Surg Oncol. 2015;41(10):1386-1392.

77. Penel N, Van Glabbeke M, Marreaud S, Ouali M, Blay JY, Hohenberger P. Testing new regimens in patients with advanced soft tissue sarcoma: analysis of publications from the last 10 years. Ann Oncol. 2011;22(6): $1266-1272$.

78. Bramwell V, Rouesse J, Steward W, et al. Adjuvant CYVADIC chemotherapy for adult soft tissue sarcoma-reduced local recurrence but no improvement in survival: a study of the European Organization for Research and Treatment of Cancer Soft Tissue and Bone Sarcoma Group. J Clin Oncol. 1994;12(6):1137-1149.

79. Frustaci S, Gherlinzoni F, De Paoli A, et al. Adjuvant chemotherapy for adult soft tissue sarco-mas of the extremities and girdles: results of the italian randomized cooperative trial. J Clin Oncol. 2001;19(5): $1238-1247$.

80. Gortzak E, Azzarelli A, Buesa J, et al. A randomised phase II study on neoadjuvant chemotherapy for "high-risk" adult soft-tissue sarcoma. Eur J Cancer. 2001;37(9):1096-1103.

81. Judson I, Verweij J, Gelderblom H, et al. European Organisation and Treatment of Cancer Soft Tissue and Bone Sarcoma Group. Doxorubicin alone versus intensified doxorubicin plus ifosfamide for first-line treatment of advanced or metastatic soft-tissue sarcoma: a randomised controlled phase 3 trial. Lancet Oncol. 2014;15(4):415-423.

82. Italiano A, Toulmonde M, Cioffi A, et al. Advanced well-differentiated/ dedifferentiated liposarcomas: role of chemotherapy and survival. Ann Oncol. 2012;23(6):1601-1607.

83. Martin-Liberal J, Alam S, Constantinidou A, et al. Clinical activity and tolerability of a 14-day infusional Ifosfamide schedule in soft-tissue sarcoma. Sarcoma. 2013;2013:868973.

84. Maki RG, Wathen JK, Patel SR, et al. Randomized phase II study of gemcitabine and docetaxel compared with gemcitabine alone in patients with metastatic soft tissue sarcomas: results of sarcoma alliance for research through collaboration study 002 [corrected]. J Clin Oncol. 2007;25(19):2755-2763.

85. Petek BJ, Loggers ET, Pollack SM, Jones RL. Trabectedin in soft tissue sarcomas. Mar Drugs. 2015;13(2):974-983.

86. D'Incalci M, Galmarini CM. A review of trabectedin (ET-743): a unique mechanism of action. Mol Cancer Ther. 2010;9(8):2157-2163.

87. D'Incalci M, Frapolli R, Germano G, Allavena P. New activities for the anti-tumor agent trabectedin: taking two birds with one stone. Oncotarget. 2013;4(4):496-497.

88. Germano G, Frapolli R, Belgiovine C, et al. Role of macrophage targeting in the antitumor activity of trabectedin. Cancer Cell. 2013;23(2): 249-262.

89. D'Incalci M, Badri N, Galmarini CM, Allavena P. Trabectedin, a drug acting on both cancer cells and the tumour microenvironment. $\mathrm{Br} J$ Cancer. 2014;111(4):646-650.

90. Forni C, Minuzzo M, Virdis E, et al. Trabectedin (ET-743) promotes differentiation in myxoid liposarcoma tumors. Mol Cancer Ther. 2009;8(2):449-457.

91. Di Giandomenico S, Frapolli R, Bello E, et al. Mode of action of trabectedin in myxoid liposarcomas. Oncogene. 2014;33(44):5201-5210.

92. Dossi R, Frapolli R, Di Giandomenico S, et al. Antiangiogenic activity of trabectedin in myxoid liposarcoma: involvement of host TIMP-1 and TIMP-2 and tumor thrombospondin-1. Int J Cancer. 2015;136(3): 721-729. 
93. Uboldi S, Calura E, Beltrame L, et al. A systems biology approach to characterize the regulatory networks leading to trabectedin resistance in an in vitro model of myxoid liposarcoma. PLoS One. 2012; 7(4):e35423.

94. Blay JY, Leahy MG, Nguyen BB, et al. Randomised phase III trial of trabectedin versus doxorubicin-based chemotherapy as first-line therapy in translocation-related sarcomas. Eur J Cancer. 2014;50(6):1137-1147.

95. Demetri GD, Chawla SP, von Mehren M, et al. Efficacy and safety of trabectedin in patients with advanced or metastatic liposarcoma or leiomyosarcoma after failure of prior anthracyclines and ifosfamide: results of a randomized phase II study of two different schedules. J Clin Oncol. 2009;27(25):4188-4196.

96. Samuels BL, Chawla S, Patel S, et al. Clinical outcomes and safety with trabectedin therapy in patients with advanced soft tissue sarcomas following failure of prior chemotherapy: results of a worldwide expanded access program study. Ann Oncol. 2013;24(6):1703-1709.

97. Gronchi A, Bui BN, Bonvalot S, et al. Phase II clinical trial of neoadjuvant trabectedin in patients with advanced localized myxoid liposarcoma. Ann Oncol. 2012;23(3):771-776.

98. Grosso F, Sanfilippo R, Virdis E, et al. Trabectedin in myxoid liposarcomas (MLS): a long-term analysis of a single-institution series. Ann Oncol. 2009;20(8):1439-1444.

99. Demetri GD, von Mehren M, Jones RL, et al. Efficacy and safety of trabectedin or dacarbazine for metastatic liposarcoma or leiomyosarcoma after failure of conventional chemotherapy: results of a phase iii randomized multicenter clinical trial. J Clin Oncol. 2015;34(8): 786-793.

100. Kawai A, Araki N, Sugiura H, et al. Trabectedin monotherapy after standard chemotherapy versus best supportive care in patients with advanced, translocation-related sarcoma: a randomised, open-label, phase 2 study. Lancet Oncol. 2015;16(4):406-416.

101. Funahashi Y, Okamoto K, Adachi Y, et al. Eribulin mesylate reduces tumor microenvironment abnormality by vascular remodeling in preclinical human breast cancer models. Cancer Sci. 2014;105(10):1334-1342.

102. Yoshida T, Ozawa Y, Kimura T, et al. Eribulin mesilate suppresses experimental metastasis of breast cancer cells by reversing phenotype from epithelial-mesenchymal transition (EMT) to mesenchymalepithelial transition (MET) states. Br J Cancer. 2014;110(6): 1497-1505

103. Dybdal-Hargreaves NF, Risinger AL, Mooberry SL. Eribulin mesylate: mechanism of action of a unique microtubule-targeting agent. Clin Cancer Res. 2015;21(11):2445-2452.

104. Cortes J, O’Shaughnessy J, Loesch D, et al. EMBRACE (Eisai Metastatic Breast Cancer Study assessing physician's choice versus E7389) investigators. Eribulin monotherapy versus treatment of physician's choice in patients with metastatic breast cancer (EMBRACE): a phase 3 open-label randomised study. Lancet. 2011;377(9769):914-923.

105. Schöffski P, Ray-Coquard IL, Cioffi A, et al. European Organisation for Research and Treatment of Cancer (EORTC) Soft Tissue and Bone Sarcoma Group (STBSG). Activity of eribulin mesylate in patients with soft-tissue sarcoma: a phase 2 study in four independent histological subtypes. Lancet Oncol. 2011;12(11):1045-1052.

106. Schöffski P, Maki RG, Italiano A, et al. Randomized, open-label, multicenter, phase III study of eribulin versus dacarbazine in patients (pts) with leiomyosarcoma (LMS) and adipocytic sarcoma (ADI). ASCO Annual Meeting 2015. J Clin Oncol. 2015;33(suppl; abstr LBA10502).

107. Schöffski P, Chawla S, Maki RG, et al. Eribulin versus dacarbazine in previously treated patients with advanced liposarcoma or leiomyosarcoma: a randomised, open-label, multicentre, phase 3 trial. Lancet. 2016;387(10028):1629-1637.

108. Shor AC, Agresta SV, D'Amato GZ, Sondak VK. Therapeutic potential of directed tyrosine kinase inhibitor therapy in sarcomas. Cancer Control. 2008;15(1):47-54.

109. Hamberg P, Verweij J, Sleijfer S. (Pre-)clinical pharmacology and activity of pazopanib, a novel multikinase angiogenesis inhibitor. Oncologist. 2010;15(6):539-547.
110. Sleijfer S, Ray-Coquard I, Papai Z, et al. Pazopanib, a multikinase angiogenesis inhibitor, in patients with relapsed or refractory advanced soft tissue sarcoma: a phase II study from the European organisation for research and treatment of cancer-soft tissue and bone sarcoma group (EORTC study 62043). J Clin Oncol. 2009;27(19):3126-3132.

111. Ahmad T, Eisen T. Kinase inhibition with BAY 43-9006 in renal cell carcinoma. Clin Cancer Res. 2004;10(18):6388-6392.

112. von Mehren M, Rankin C, Goldblum JR, et al. Phase 2 Southwest Oncology Group-directed intergroup trial (S0505) of sorafenib in advanced soft sarcomas. Cancer. 2012;118(3):770-776.

113. Maki RG, D'Adamo DR, Keohan ML, et al. Phase II study of sorafenib in patients with metastatic or recurrent sarcomas. J Clin Oncol. 2009; 27(19):3133-3140.

114. Demetri GD, van Oosterom AT, Garrett CR, et al. Efficacy and safety of sunitinib in patients with advanced gastrointestinal stromal tumour after failure of imatinib: a randomised controlled trial. Lancet. 2006;368(9544):1329-1338.

115. Mahmood ST, Agresta S, Vigil CE, et al. Phase II study of sunitinib malate, a multitargeted tyrosine kinase inhibitor in patients with relapsed or refractory soft tissue sarcomas. Focus on three prevalent histologies: leiomyosarcoma, liposarcoma and malignant fibrous histiocytoma. Int J Cancer. 2011;129(8):1963-1969.

116. Schuetze SM, Wathen JK, Lucas DR, et al. SARC009: Phase 2 study of dasatinib in patients with previously treated, high-grade, advanced sarcoma. Cancer. 2016;122(6):868-874.

117. Vassilev LT, Vu BT, Graves B, et al. In vivo activation of the p53 pathway by small-molecule antagonists of MDM2. Science. 2004; 303(5659):844-848.

118. Müller CR, Paulsen EB, Noordhuis P, Pedeutour F, Saeter G, Myklebost O. Potential for treatment of liposarcomas with the MDM2 antagonist Nutlin-3A. Int J Cancer. 2007;121(1):199-205.

119. Singer S, Socci ND, Ambrosini G, et al. Gene expression profiling of liposarcoma identifies distinct biological types/subtypes and potential therapeutic targets in well-differentiated and dedifferentiated liposarcoma. Cancer Res. 2007;67(14):6626-6636.

120. Ray-Coquard I, Blay JY, Italiano A, et al. Effect of the MDM2 antagonist RG7112 on the P53 pathway in patients with MDM2-amplified, well-differentiated or dedifferentiated liposarcoma: an exploratory proof-of-mechanism study. Lancet Oncol. 2012;13(11):1133-1140.

121. Italiano A, Bianchini L, Gjernes E, et al. Clinical and biological significance of CDK4 amplification in well-differentiated and dedifferentiated liposarcomas. Clin Cancer Res. 2009;15(18): 5696-5703.

122. Lee S, Park H, Ha SY, et al. CDK4 amplification predicts recurrence of well-differentiated liposarcoma of the abdomen. PLoS One. 2014;9(8):e99452.

123. Luke JJ, D’Adamo DR, Dickson MA, et al. The cyclin-dependent kinase inhibitor flavopiridol potentiates doxorubicin efficacy in advanced sarcomas: preclinical investigations and results of a phase I doseescalation clinical trial. Clin Cancer Res. 2012;18(9):2638-2647.

124. Benson C, White J, De Bono J, et al. A phase I trial of the selective oral cyclin-dependent kinase inhibitor seliciclib (CYC202; R-Roscovitine), administered twice daily for 7 days every 21 days. Br J Cancer. 2007; 96(1):29-37.

125. Dickson MA, Tap WD, Keohan ML, et al. Phase II trial of the CDK4 inhibitor PD0332991 in patients with advanced CDK4-amplified well-differentiated or dedifferentiated liposarcoma. J Clin Oncol. 2013;31(16):2024-2028

126. Patnaik A, Rosen LS, Tolaney SM, et al. Clinical activity of LY2835219, a novel cell cycle inhibitor selective for CDK4 and CDK6, in patients with metastatic breast cancer. Paper presented at: the American Association of Cancer Research Annual Meeting; April 2014; San Diego, CA (abstr CT232).

127. Infante JR, Shapiro GI, Witteveen JF, et al. Phase 1 multicenter, open label, dose-escalation study of LEE011, an oral inhibitor of cyclin-dependent kinase $4 / 6$, in patients with advanced solid tumors or lymphomas. Mol Cancer Ther. 2013;12(suppl 11, abstr A276). 
128. Tontonoz P, Singer S, Forman BM, et al. Terminal differentiation of human liposarcoma cells induced by ligands for peroxisome proliferator-activated receptor gamma and the retinoid $\mathrm{X}$ receptor. Proc Natl Acad Sci U S A. 1997;94(1):237-241.

129. Demetri GD, Fletcher CD, Mueller E, et al. Induction of solid tumor differentiation by the peroxisome proliferator-activated receptorgamma ligand troglitazone in patients with liposarcoma. Proc Natl Acad Sci U S A. 1999;96(7):3951-3956.

130. Debrock G, Vanhentenrijk V, Sciot R, Debiec-Rychter M, Oyen R, Van Oosterom A. A phase II trial with rosiglitazone in liposarcoma patients. Br J Cancer. 2003;89(8):1409-1412.

131. Pishvaian MJ, Marshall JL, Wagner AJ, et al. A phase 1 study of efatutazone, an oral peroxisome proliferator-activated receptor gamma agonist, administered to patients with advanced malignancies. Cancer. 2012;118(21):5403-5413.

132. Chow WA, Guo S, Valdes-Albini F. Nelfinavir induces liposarcoma apoptosis and cell cycle arrest by upregulating sterol regulatory element binding protein-1. Anticancer Drugs. 2006;17(8):891-903.
133. Pan J, Mott M, Xi B, et al. Phase I study of nelfinavir in liposarcoma. Cancer Chemother Pharmacol. 2012;70(6):791-799.

134. Dickson MA, D’Adamo DR, Keohan ML, et al. Phase II trial of gemcitabine and docetaxel with bevacizumab in soft tissue sarcoma. Sarcoma. 2015;2015:532478.

135. Ha HT, Griffith KA, Zalupski MM, et al. Phase II trial of cetuximab in patients with metastatic or locally advanced soft tissue or bone sarcoma. Am J Clin Oncol. 2013;36(1):77-82.

136. Hwang JA, Yang HM, Hong DP, et al. Gankyrin is a predictive and oncogenic factor in well-differentiated and dedifferentiated liposarcoma. Oncotarget. 2014;5(19):9065-9078.

137. Patnaik A, Kang SP, Rasco D, et al. Phase I study of pembrolizumab (mk-3475; anti-pd-1 monoclonal antibody) in patients with advanced solid tumors. Clin Cancer Res. 2015;21(19):4286-4293.
OncoTargets and Therapy

\section{Publish your work in this journal}

OncoTargets and Therapy is an international, peer-reviewed, open access journal focusing on the pathological basis of all cancers, potential targets for therapy and treatment protocols employed to improve the management of cancer patients. The journal also focuses on the impact of management programs and new therapeutic agents and protocols on

\section{Dovepress}

patient perspectives such as quality of life, adherence and satisfaction The manuscript management system is completely online and includes a very quick and fair peer-review system, which is all easy to use. Visit http://www.dovepress.com/testimonials.php to read real quotes from published authors. 